\title{
Suppression of activated Let-60 Ras protein defines a role of Caenorhabditis elegans Sur-1 MAP kinase in vulval differentiation
}

\author{
Yan Wu and Min Han ${ }^{1}$ \\ Department of Molecular, Cellular, and Developmental Biology, University of Colorado at Boulder, Boulder, Colorado \\ 80309-0347 USA
}

The let-60 ras gene of Caenorhabditis elegans is one of the key players in a signal transduction pathway that controls the choice between vulval and epidermal differentiation in response to extracellular signals. To identify components acting downstream of let-60 ras in the vulval signaling pathway, we have identified a reduction-of-function mutation in the sur-1 gene that completely suppresses the multivulva phenotype of a hyperactive let-60 ras mutation. About $10 \%$ of animals homozygous for the sur-1 mutation also display a specific and intriguing vulval cell lineage defect. In addition, the sur-1 mutation results in a cold-sensitive egg-laying defective phenotype and a partial larval lethal phenotype. We have cloned the sur-1 gene by DNA-mediated transformation and have shown that it encodes a protein similar in overall structure to mammalian MAP kinases (ERKs). The functional homology between Sur-1 MAP kinase and mammalian MAP kinases was also demonstrated by the ability of a rat ERK2 kinase to rescue the sur-1 mutant phenotypes. Genetic double-mutant analyses place sur-1 downstream of let-60 ras but upstream of lin-1 in the vulval signaling pathway. Our results provide further evidence for the extreme conservation of Ras-mediated signaling pathway between worms and humans and for the function of MAP kinases in cell signaling processes that control cell differentiation and animal development.

[Key Words: MAP kinase; sur-1; ras; C. elegans; vulval development; signal transduction]

Received November 2, 1993; revised version accepted December 8, 1993.

Development of the hermaphrodite vulva in the nematode Caenorhabditis elegans is initiated by intercellular signaling (for review, see Horvitz and Sternberg 1991). Prior to vulval induction, a set of six ectodermal precursor cells (P3.p-P8.p) have the same developmental potential. According to the current model, the combination of an inhibitory signal from the epidermis and an inductive signal from the gonadal anchor cell induces three of the six precursor cells (P5.p-P7.p) to generate vulval tissue. The other three cells (P3.p, P4.p, and P8.p) generate nonspecific epidermis (Fig. 1A). Genetic and molecular studies have defined roles for several cell signaling proteins in vulval induction (see Fig. 6, below). For example, the let-60 gene encodes a Ras protein that acts downstream of let-23 (receptor tyrosine kinase) and sem-5 (a protein with $\mathrm{SH} 2$ and $\mathrm{SH} 3$ domains| to control vulval cell fates (Aroian et al. 1990; Han and Sternberg 1990; Clark et al. 1992). Loss-of-function mutations in let-60 ras result in fewer than three vulval precursor cells (VPCs) to generate vulval cells (vulvaless or Vul),

${ }^{1}$ Corresponding author. whereas gain-of-function (gf) let-60 ras mutations result in more than three VPCs to generate vulval cells (multivulva or Muv) (Beitel et al. 1990; Han et al. 1990). The lin-45 gene, which encodes a homolog of the mammalian Raf kinases, appears to act after let-60 ras but upstream of lin-1 in the signaling pathway (Han et al. 1993).

Well-established genetics in C. elegans provided us with an opportunity to identify new components in the Ras-mediated cell signaling pathway and to analyze their role in developmental pattern formation. Here, we report a genetic and molecular analysis of the sur-1 gene. The genetic analysis of a mutation in the sur-1 gene suggests that it is likely to act downstream of the let-60 ras gene to affect the vulval signaling process. The molecular analysis of the sur-1 gene demonstrates that it encodes a protein structurally and functionally homologous to mammalian p42 MAP kinases. Our present results provide in vivo evidence for the function of MAP kinases in receptor tyrosine kinase/Ras-mediated cell signaling processes that control cell differentiation and proliferation. Similar and complementary results on the same $C$. elegans MAP kinase gene have been obtained independently by Lackner et al. (this issue). 
A wild type
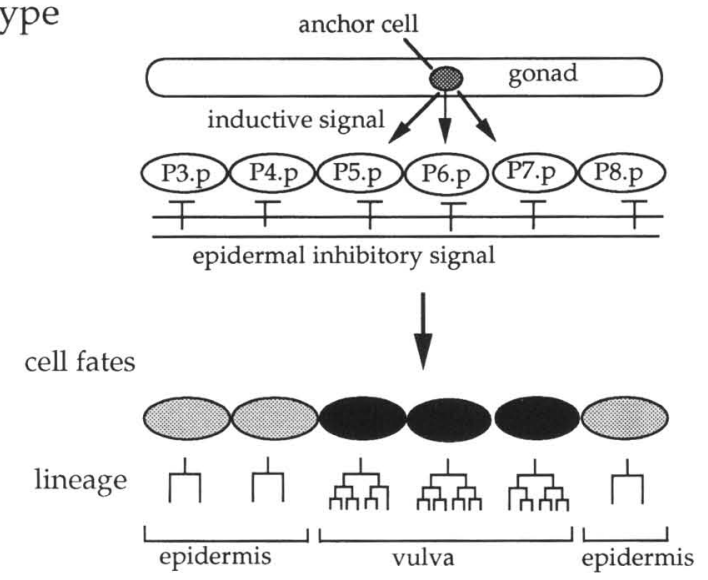

B let-60(gf) mutant

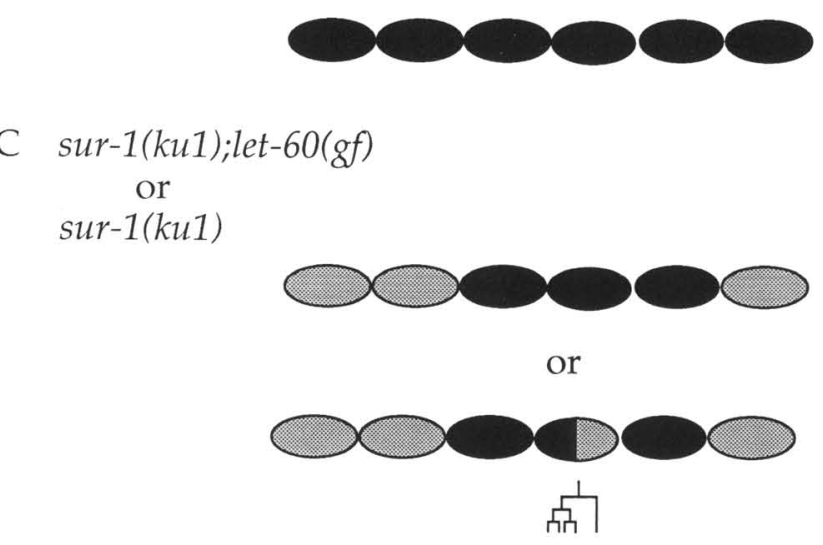

Figure 1. Vulval cell fate specification in wild-type and mutant animals. $(A)$ Vulval cell fate specification in wild-type animals. The six multipotent (VPCs, P3.p-P8.p; depicted as open ovals) are located just ventral to the gonad. According to the current model, an inhibitory signal, possibly from the epidermis, prevents vulval differentiation (Herman and Hedgecock 1990). However, an inductive signal from the anchor cell in the gonad induces the three nearby VPCs to adopt vulval cell fates (solid ovals) (Horvitz and Sternberg 1991; Hill and Sternberg 1992). These induced cells divide three rounds, and the progeny generate vulval tissue. The other three precursor cells divide only once and then become part of a large epidermal syncytium (shaded ovals). The lineages of each VPC are depicted (Sulston and Horvitz 1977). P3.p does not divide in 50\% of the wild-type hermaphrodites. (B) Vulval cell fates in let-60/n1046 gf) mutant animals. The activated let-60 ras mutation results in more than three of the six VPCs (often all six cells as depicted) differentiating into vulval cells (Muv). The average vulval cell differentiation in the mutant is $\sim 158 \%$ of that observed in wild type (Han et al. 1990, 1993). (C) Vulval cell fates in sur-1(ku1); let-60(n1046 gf) double-mutant animals or in sur-1(ku1) single-mutant animals. The majority of animals of either genotype have a wild-type vulval cell lineage as indicated by the top drawing. Ten percent $(2 / 20)$ of the sur-1 single mutants and $10 \%(3 / 30)$ of the sur-1(ku1); let-60(n1046 gf) double mutants have defective vulval cell differentiation as indicated in the bottom drawing. One of the two daughter cells of P6.p fails to divide further and differentiate into vulval tissue (half shaded/half solid ovall, whereas the two adjacent VPCs are normal, resulting in two small ventral protrusions. The defective P6.p daughter cell can be at either the left or right side of the normal daughter cell.

\section{Results}

A sur-1 mutation suppresses an activated let-60 ras mutation

To identify additional factors acting downstream of Let60 Ras in the signal transduction pathway that induce vulval development, we conducted screens to isolate mutations that suppress the Muv phenotype of an activated let-60 ras mutation [let-60(n1046 gf), a point mutation that changes Gly-13 to Glu-13 (Beitel et al. 1990)]. The sur-1 (suppressor of ras mutation/gene is defined by one of these suppressor mutations (ku1). The Muv phenotype of the activated ras mutation is completely suppressed in animals homozygous for ku1 and let-60(n1046 gf) (Figs. 1 and 2; Table 1). Animals homozygous for ku1 also display incompletely penetrant egg-laying-defective (Egl) and partial larval lethal phenotypes (Table 2). The dying ku1 homozygous animals arrest at different larval stages. The earliest arrest appears to be at the Ll larval stage based on the size of the gonad and the presence of L1-specific alae in 12 dead larvae observed by Nomarski microscopy. This partial lethal phenotype suggests that the sur- 1 gene has an essential function during or before the $\mathrm{L} 1 \mathrm{larval}$ stage. The larval lethality is suppressed by the presence of let-60(n1046 gf) in the sur-1(ku1); let60(n1046 gf) double mutants.

The Egl phenotype of ku1 homozygotes is cold sensitive: About $71 \%$ of the animals are $\mathrm{Egl}$ at $15^{\circ} \mathrm{C}$, whereas only $32 \%$ are Egl at $20^{\circ} \mathrm{C}$ (Table 2 ). The penetrance of the Egl phenotype remains essentially the same in sur1(ku1); let-60(n1046) double-mutant animals (Table 1). The Egl phenotype is partly the consequence of a disruption of vulval cell differentiation because $\sim 10 \%$ of $k u 1$ homozygotes show incomplete vulval cell differentiation. It is interesting to note that in all animals with a vulval cell lineage defect, only one of the two P6.p daughter cells failed to divide further to generate vulval cells whereas the two adjacent VPCs were induced normally, resulting in two small ventral protrusions (Figs. $1 \mathrm{C}$ and $2 \mathrm{C}$. Two different cell fates associated with the daughters of individual VPCs (a "hybrid" lineage) were observed previously for the let-23, let-60, and lin-45 genes (Aroian and Sternberg 1991; M. Han and P.W. Sternberg, unpubl.). These hybrid lineages suggest that the VPCs are not irreversibly committed to vulval cell fates before their first round of cell division. However, only in the sur-1 mutant, the hybrid lineage is exclusively associated with P6.p, suggesting that P6.p, which is located closest to the anchor cell, is more sensitive than other VPCs to the activity change in sur-1. Because the Egl phenotype of ku1 homozygotes is more severe than would be accounted for by the defect in the vulval cell lineage, the ku1 mutation is likely to disrupt some other aspects of the C. elegans egg-laying system.

The suppression of the Muv phenotype of an activated let-60 ras mutation, the Egl phenotype, and the partial 

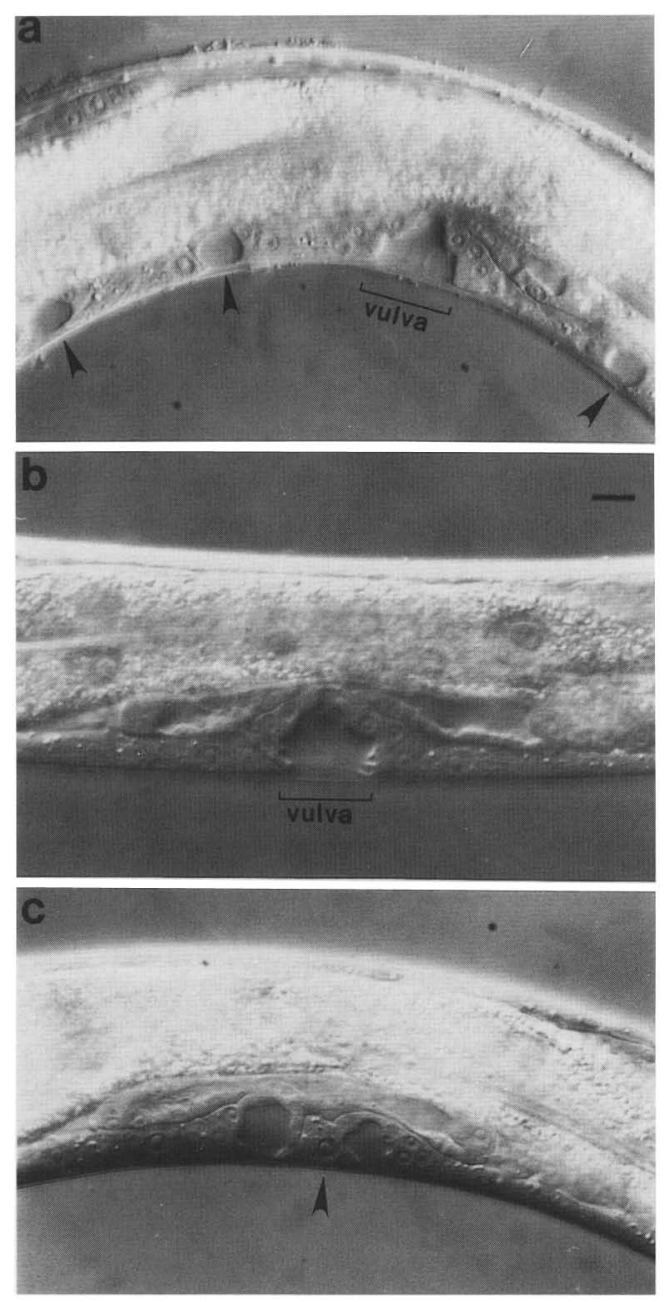

Figure 2. Nomarski photomicrographs showing vulval cell differentiations in C. elegans mutant hermaphrodites. All three animals are at the early L4 larval stage and magnified $100 \times\langle b|$. Scale bar, $8 \mu \mathrm{m}$. (a) A let-60(n1046 gf) animal. Normal vulval cell differentiation is indicated by normal divisions and morphogenesis of cells derived from P5.p, P6.p, and P7.p (bracket). The let 60 mutation also causes extra vulval cell differentiation and a Muv phenotype: Extra cell divisions and morphogenesis associated with the progeny of P3.p, P4.p, and P8.p are indicated by the three extra pseudovulval invaginations (arrowheads). The average vulval cell differentiation of the let-60(n1046 gf) mutants is $\sim 158 \%$ (Table 1 ), or an average of $\sim 1.7$ extra cells differentiate to vulval tissues per animal. (b) A sur-1(ku1); let60(n1046 $\mathrm{gf}$ ) animal. In this animal, the Muv phenotype is completely suppressed (no extra vulval cell differentiations). (c) A sur-1(ku1) animal with a phenotype observed in $\sim 10 \%$ of the mutant animals. The arrowhead indicates the posterior daughter cell of P6.p (P6.pp) that failed to go through further cell division and vulval differentiation. Vulval differentiation and morphogenesis of cells derived from P6.pa and P5.p is indicated by the invagination to the left of the undivided P6.pp cell, and vulval differentiation and morphogenesis of cells derived from P7.p is indicated by the invagination to the right of the undivided P6.pp cell.
Table 1. Genetic interactions of sur-1 with let-60, lin-15, or lin-1

\begin{tabular}{|c|c|c|}
\hline Genotype $^{a}$ & $\begin{array}{l}\text { Percent } \\
\text { Muv }(n)^{\mathrm{b}}\end{array}$ & $\begin{array}{l}\text { Percent } \\
\text { vulval } \\
\text { differentiation } \\
\langle n\rangle^{\mathrm{c}}\end{array}$ \\
\hline Wild type & 0 & 100 \\
\hline sur-1/sur-1 & $0(200)$ & $98(20)$ \\
\hline$+/+;$ let $-60($ gf $) /$ let $-60($ gf $)(1)$ & $95(150)$ & 158 \\
\hline$+/+;$ let $-60(g f) / \operatorname{let}-60(\mathrm{gf})(2)$ & $80(187)$ & N.D. \\
\hline sur-1/+; let-60(gf)/let-60(gf) & $64(255)$ & N.D. \\
\hline sur-1/sur- $1 ;$ let-60(gf)/let-60(gf) & $0(300)$ & $98(30)$ \\
\hline$+++; \operatorname{let}-60(g f) \operatorname{let}-60(g f)$ & $94(427)$ & N.D. \\
\hline sur- $1 /+/+;$ let $-60(g f) / 1$ et $-60(g f)$ & $96(49)$ & N.D. \\
\hline sur-1/sur-1/+; let-60(gf)/let-60(gf) & $90 \mid 575)$ & N.D. \\
\hline lin-15/lin-15 & $99(100)$ & $200(12)$ \\
\hline sur-1/sur- $1 ;$ lin-15/lin-15 & $5(100)$ & $101(22)$ \\
\hline $\operatorname{lin}-1 / \operatorname{lin} 1$ & $100(100)$ & 165 \\
\hline sur-1/sur-1; lin-1/lin1 & $100(200)$ & $167(10)$ \\
\hline
\end{tabular}

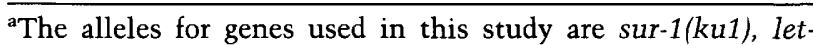
60(n1046 gf), lin-15(n765), and lin-1(e1275). (+) A wild-type copy of sur-1. A duplication covering sur-1 $(s D p 3)$ provided the additional wild-type copy of sur-1. (gf) Gain-of-function mutations. let-60(n1046 gf) results in a substitution of glutamate for glycine in codon 13 of let-60 ras (Beitel et al. 1990). Two strains were used to examine the Muv phenotype of let-60(n1046gf) homozygotes. The first is let-60(gf)/let-60(gf) (1), and the second is unc-79 $+/+$ dpy-17; let-60(gf)/let-60(gf) (2). The complete genotypes of other strains listed are + sur-1 dpy-17/unc-79+ + ; let-60(gf) let-60(gf) [for sur-1/+; let-60(gf)/let-60(gf)], sur-1/ sur-1; let-60(gf)/let-60(gf), dpy-17 ncl-1 unc-36/dpy-17 ncl-1 unc-36; let-60(gf)/let-60(gf); sDp3 [for $+/+/+$; let-60(gf)/let$60(\mathrm{gf})$ ), sur-1 dpy-17+ +/+dpy-17 ncl-1 unc-36; let-60(gf)/let$60(g f) ; s D p 3$ [for sur-1/+/+ ; let-60(gf)/let-60(gf)], sur-1 dpy-17/ sur-1 dpy-17; let-60(gf)/let-60(gf); sDp3 [for sur-1/sur-1/+; let60(gf)/let-60(gf)], lin-15/lin-15, sur-1/sur-1; lin-15/lin-15, lin-1/ lin-1, and sur-1/sur-1; lin-1 dpy-20/lin-1 dpy-20 [for sur-1/sur-1; lin-1/lin-1].

bercentage of animals having a Muv phenotype observed as multiple ventral protrusions under a dissection microscope (Ferguson and Horvitz 1985). The Muv phenotype of let60(n1046 gf), lin-15(n765 lf), or lin-1(e1275 lf) has been described previously (Beitel et al. 1990; Ferguson and Horvitz 1985; Han et al. 1990, 1993).

'Percentage of vulval precursor cells (P3.p-P8.p) that differentiate into vulval cells relative to wild type (Han et al. 1990). The numbers of animals examined in this study by Nomarski microscopy is indicated $(n)$. Percentage of vulval differentiation for the let-60 and lin-1 single mutants was described previously (Han et al. 1993). Among the 20 sur-1(ku1) mutant animals, 2 had $83 \%$ vulval differentiation (see Fig. 1) and the rest had $100 \%$ vulval differentiation. Among the 30 sur-1; let- 60 double mutant animals, 3 had $83 \%$ vulval differentiation and the rest had $100 \%$. Among the 22 sur-1; lin-15 double mutant animals, 1 had $133 \%$ vulval differentiation, 1 had $83 \%$ vulval differentiation, and the rest had $100 \%$ vulval differentiation. The 10 sur- 1 ; lin-1 double mutant animals had vulval differentiation ranging from $150 \%$ to $183 \%$. The data indicate that the sur-1(ku1) mutation suppresses the multivulva phenotype of $\operatorname{lin}-15(n 765)$ and let60 (n1046 gf) animals but not that of lin-1(e1275) animals. 
Table 2. Egl and larval lethal phenotypes of sur-1(ku1)

\begin{tabular}{lcccc}
\hline & \multicolumn{2}{c}{ Percent Egl $(n)^{\mathrm{b}}$} & \multicolumn{2}{c}{${\text { Percent lethality }(n)^{\mathrm{c}}}^{\mathrm{n}}$} \\
\cline { 2 - 5 } Genotype $^{\mathrm{a}}$ & $20^{\circ}$ & $15^{\circ}$ & $20^{\circ}$ & $15^{\circ}$ \\
\hline sur-1(ku1) & $32(200)$ & $71(200)$ & $7.5(200)$ & $7(200)$ \\
sur-1(ku1); let-60(n1046 gf) & $38(180)$ & $77(200)$ & $0(400)$ & $0(400)$ \\
sur-1(ku1)/+ & $1(200)$ & $1(270)$ & N.D. & N.D. \\
sur-1(ku1)/sur-1(ku1)/+ & $1(795)$ & $1(255)$ & N.D. & N.D. \\
\hline
\end{tabular}

${ }^{a}$ The complete genotype for sur-1(ku1)/ + is sur-1(ku1) dpy-17(e164)/qC1, and the complete genotype for sur-1(ku1)/sur-1(ku1)/+ is sur-1(ku1) dpy-17/sur-1(ku1) dpy-17; sDp3.

bPercentage of hermaphrodites that are Egl.

'Percentage of animals that die before reaching adulthood.

larval lethality of sur-1(ku1) are most likely to be the result of reduction of the sur-1 gene activity. sur$1(k u 1) /+$ or sur-1(ku1)/sur-1(ku1)/ + animals are egglaying competent (Table 2), indicating that the Egl phenotype of ku1 is recessive. The additional wild-type copy of sur-1 in sur-1(ku1)/sur-1(ku1)/ + animals is provided by a free duplication (sDp3; see Materials and methods). The Muv phenotype of let-60(gf), however, appears to be more sensitive to activity change in the sur-1 gene. A strain of sur-1(ku1)/+; let-60(n1046 gf) is $\sim 64 \%$ Muv, which is less than that of the let-60(n1046 gf) single mu$\operatorname{tant}(>80 \%$, Table 1$)$. Such a difference may be the result of weak dominance (dominant suppression of the Muv phenotype) of sur-1(ku1). However, it is unlikely that such weak dominance could be attributed to a gain-offunction nature of sur-1(ku1) because sur-1(ku1)/sur$1(\mathrm{ku1}) /+$; let-60(gf) animals have a higher Muv percentage than that of sur-1(ku1)/ + let-60(gf) animals $(90 \%$ compared with $64 \%$; Table 1). Therefore, sur-1(ku1) is likely to be a partial loss-of-function mutation, and partial suppression of the let-60(gf) Muv phenotype in sur$1(\mathrm{ku} 1) /+$; let-60(n1046 gf) strains could be the result of a reduction $(<50 \%)$ of sur-1 gene activity in the heterozygous strain. sur-1(ku1)/deficiency animals appear to be lethal and/or sterile on the basis of the following experiment. sur-1(ku1) dpy-17/qC1; him-5 males were crossed with $n D f 11+/$ unc-79 dpy-17 hermaphrodites. Among 179 fertile, non-Dpy, non-Unc cross progeny picked, none were sur-1(ku1) $d p y-17 / n D f 11+$ (see Materials and methods). As a control, $d p y-17 / q C 1$; him-5 males were crossed with $n D f 11+/$ unc-79 dpy-17 hermaphrodites. Among 39 non-Dpy, non-Unc cross progeny obtained, 16 were $+d p y-17 / n D f 11+$ animals. Therefore, it is likely that a null mutation in sur- 1 would result in recessive lethality at an early larval stage and thus would not be recovered by screening for suppressors of let-60(n1046 gf). Recessive lethality in early larval stages is also the null phenotype of a number of other genes acting in the vulval signaling pathway (Beitel et al. 1990; Han et al. 1990; Aroian and Sternberg 1991; Clark et al. 1992; Hill and Sternberg 1992). It is possible that all of these genes, including sur-1, act together in a cell signaling pathway controlling early developmental events.

Molecular cloning of the sur-1 gene

We first genetically mapped the ku1 mutation to a region between $d p y-27$ and $d p y-17$ on the left arm of chromosome III (Table 3; Fig. 3A). sur-1 was then mapped with respect to a restriction fragment length polymorphism (RFLP) generated by a transposon insertion, MJ\#NEC2 (Nishiwaki et al. 1993), between unc-79 and dpy-17 (Fig. 3B). This placed the sur-1 gene to the left of the RFLP in a region of the physical map that has been well established with cosmids and YAC DNAs (A. Coulson and J. Sulston, pers. comm.).

Cosmids containing the sur-1 gene were identified following the results of microinjection transformation with cosmids lying between $d p y-27$ and MJ\#NEC2. Plasmid pRF4, containing dominant rol-6 mutant DNA, was coinjected as a marker (Mello et al. 1991). Because the most penetrant phenotype caused by the sur-1 mutation is the complete suppression of the Muv phenotype of let-60(n1046 gf), the best way to score for the rescue of the sur-1 mutant phenotype by DNA clones containing the wild-type sur-1 gene is to score the reversion of the suppression phenotype. We used sur-1(ku1); let60 (n1046 gf) as the host strain for microinjections and score the rescue of the sur-1 mutant phenotype by the reappearance of the Muv phenotype caused by let60(n1046 gf). We determined that four overlapping cosmids, $\mathrm{F} 43 \mathrm{Cl}$, C49A12, DD7, and $\mathrm{R} 74$, were each able to rescue the sur-1 mutant phenotype (Fig. 3B). The sur-1 (ku1); let-60(n1046 gf) strain is sensitive to the dosage of extrachromosomal DNA that contains the sur-1 gene; rescued transgenic animals were often sick, and injection of high concentrations $(>30 \mathrm{ng} / \mu \mathrm{l})$ of the DNA clones appeared to cause a lethal phenotype (no transgenic progeny obtained; data not shown). However, injection of a positive clone (C49A12) into wild-type animals did not cause a Muv phenotype (data not shown).

The overlapping region of the four cosmids containing the sur-1 gene was further characterized by restriction digestions, Southern analysis, subcloning, and microinjection. We determined that a combination of two overlapping genomic fragments (subclones pYW2 and pYW7) rescued the sur-1(ku1) mutant phenotype, although neither plasmid alone gave a positive result (Fig. 3B). Obtaining a functional gene through homologous recombination of truncated gene fragments during microinjection transformation has been well established in $C$. elegans (e.g., Aroian et al. 1990; Mello et al. 1991). According to our later analysis, pYW2 and pYW7 do not 
Table 3. Genetic three-point mapping of sur-1(kul) on chromosome III

\begin{tabular}{|c|c|c|}
\hline $\begin{array}{l}\text { Genotype of } \\
\text { heterozygotes }^{a}\end{array}$ & $\begin{array}{l}\text { Phenotype of } \\
\text { recombinants }^{b}\end{array}$ & $\begin{array}{l}\text { Recombinants with } \\
\text { sur-1/total recombinants }\end{array}$ \\
\hline$\frac{\text { sur }-1++}{+ \text { dpy-17 unc-32 }}$ & Unc non-Dpy & $21 / 21$ \\
\hline$\frac{+ \text { sur-1 }+}{\text { unc-93 }+d p y-17}$ & Dpy non-Unc & $4 / 21$ \\
\hline$\frac{+ \text { sur- } 1+\text { unc-32 }}{\text { unc-79 }+ \text { dpy-17 }}$ & Dpy non-Unc & $6 / 6$ \\
\hline$\frac{+ \text { sur- } 1+}{d p y-27+u n c-32}$ & Unc non-Dpy & $15 / 17$ \\
\hline unc-79 + MJ\#NEC2 dpy-17+ & Unc non-Dpy & 0/8 (8/8 with MJ\#NEC2) \\
\hline+ sur-1 + + unc-32 & Dpy non-Uuc & 13/13 (2/11 with M)\#NEC2) \\
\hline
\end{tabular}

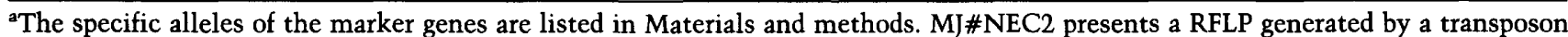
(Tc1) insertion (Nishikawi et al. 1993).

${ }^{b}$ (Unc) Uncoordinated movement; (Dpy) dumpy body shape. In the experiment listed at the bottom, Unc presents the phenotype of the unc-79 mutation. The unc-32 phenotype was not scored.

${ }^{\mathrm{C}}$ Number of recombinant animals that retain the sur-1(ku1) allele out of total recombinants homozygous for one marker gene. Determination of the presence of RFLP (MJ\#NEC2) in the recombinants is desribed in Materials and methods.

contain the first exon of the gene; however, they are likely to contain the entire coding region and part of the large first intron, which may provide some promoter activity (Figs. 3 and 4). The overlapping region of these two DNA fragments was then used as a probe to screen a cDNA library (Barstead and Waterston 1989). Six positive clones were identified from a screening of $\sim 2 \times 10^{6}$ plaques. All six cDNA clones probably encode the same protein based on their restriction maps and their $5^{\prime}$ sequences (data not shown).

\section{The sur-1 gene encodes a MAP kinase homolog}

We have obtained the sequence of one of the longer cDNA clones (Fig. 4). The predicted open reading frame can be translated into a protein of 444 amino acids, starting from the first methionine, or a protein of 376 amino acid, starting from the second methionine. The integrity of the cDNA sequence was confirmed by sequencing the relevant genomic DNA regions, including all exons and intron/exon boundaries. The overall gene structure, including the positions of all introns and exons, is indicated in Figures 3C and 4. A comparison of the protein sequence to sequences in GenBank revealed a striking amino acid sequence similarity with the MAP (mitogenactivated protein) kinase family of serine/threonine protein kinases (also known as ERKs for extracellular signalregulated kinases|. The sur-1 gene product is most closely related to the ERK2 subfamily (also known as p42 MAP kinases) (Fig. 5). The sur-1 gene product is $\sim 81 \%$ identical in amino acid sequence to human or rat ERK2 proteins in a 339-amino-acid region and $\sim 78 \%$ identical in amino acid sequence to Drosophila MAP kinase-related protein (Dm-ERK-A) in a 342-amino-acid region. All subdomains of MAP kinases, including the putative phosphorylation sites (Thr-188 and Tyr-190 for the worm protein; Thr-183 and Tyr-185 for the rat pro- tein) and the characteristic RWYR sequence located just before the APE sequence within kinase subdomain VIII, are conserved between the sur-1 gene product and the MAP kinases (Fig. 5). MAP kinases or ERKs are a family of protein kinases that have been characterized extensively as signaling intermediates in the response of eukaryotic cells to external stimuli (for review, see Cobb et al. 1991; Pelech and Sanghera 1992; Thomas 1992). The role of the sur-1 gene in the let-60 ras-mediated vulval signaling pathway provides further evidence that Rasmediated signal-transduction pathways, at least from receptor tyrosine kinases to MAP kinases, are extremely conserved between invertebrates (e.g., C. elegans and Drosophila) and vertebrates (e.g., mouse and human) (Ahn et al. 1992; Crews and Erikson 1993).

In the protein sequence translated from the cDNA sequences (Fig. 4), there are two in-frame methionines that lie upstream of the amino acids conserved with the $\mathrm{p} 42$ MAP kinases. The first ATG starts at the third nucleotide of the largest cDNA clone and is 68 codons before the second ATG. In the genomic DNA sequence, these two ATG sequences are separated by a large intron $1 \sim 12$ $\mathrm{kb})$. Because a conceptual protein starting at the second ATG has a similar amino terminus and overall size as p42 MAP kinases from other organisms and because genomic DNA without the first exon can rescue the sur1(ku1) mutant phenotype (Fig. 3), we display the protein sequence starting at the second methionine in Figure 5 with the reservation that the protein sequence might still initiate at the first methionine.

\section{Molecular lesion of sur-1(kul)}

We have also identified the molecular lesion of the sur1(ku1) mutation by sequencing the coding region and intron/exon boundaries of the mutant DNA. The mutant DNA was PCR amplified using multiple sets of oli- 
Figure 3. Molecular genetics of the sur-1 locus. (A) Genetic map position of the sur-1 gene. The sur-1 gene was genetically mapped between $d p y-27$ and $d p y-17$ on chromosome III. sur-1 was also determined to be to the right of unc- 79 by locating the right breakpoint of $y D f 10$ in the region between unc-79 and sur-1. (B) Identification of DNA clones containing the sur-1 gene. The sur-1 gene was mapped to the left of a RFLP generated by a Tc1 insertion, MJ\#NEC2 (Nishiwaki et al. 1993). To locate the sur-1 gene, all cosmids shown on the map were tested by microinjection transformation. Four of the clones were able to rescue the sur-1 mutant phenotype $(+)$; the rest of clones gave negative results $(-)$. A partial restriction map of DNA within or near the overlapping region among the four positive cosmids is also shown. The orientation of the plasmid map is inverted so that the gene is in a left to right orientation. DNA subclones of the region were also tested by microinjection transformation. Only the combination of pYW2 and pYW7 gave a positive result, indicating that the gene is located within a 13.1-kb DNA region encompassed by these plasmids. Restriction enzymes: (B) BamHI; (S) SmaI; (X) XbaI; (H) HindIII. $(C)$ Exon/intron distribution of the sur-1 gene. Seven exons ( $\square$ ) and six introns ( lines) are depicted along with their sizes in base pairs. Exon 1 is separated from exon 2 by a large intron and is not required to rescue the sur-1 mutant phenotype (injection with pYW7 plus pYW2), indicating that there is some promoter activity within the second half of intron 1. There are two putative translational start codons (ATG); one in exon 1 and the other at the beginning of exon 2 .

The two SmaI sites are the cloning sites of pYW2. (D) sur-1/ERK2 fusion gene. The ATG start codon of the rat ERK 2 cDNA was fused to the ATG codon that is at the beginning of exon 2 of sur-1. The $5^{\prime}$ end of the sur-1 sequence is located in intron 1 and is the same as the $5^{\prime}$ end of pYW2. To increase expression efficiency, the C. elegans unc-54 3' polyadenylation addition sequence (A. Fire et al. pers. comm.) was added to the $3^{\prime}$ end of the ERK2 cDNA. The fusion plasmid rescues the sur-1 mutant phenotype upon injection into a sur-1(ku1); let-60(n1046 gf) double mutant.

gonucleotide primers. The PCR products were sequenced directly. A single nucleotide change $(C \rightarrow T)$ was detected in codon 38 of the predicted nucleotide sequence, resulting in the substitution of valine for alanine (Figs. 4 and 5). Ala-38 is conserved among p42 MAP kinases and is within the Gly-Glu-Gly-Ala-Tyr-Gly motif that corresponds with the Gly-X-Gly-X-X-Gly consensus commonly found in subdomain I of many kinases as well as many nucleotide-binding proteins (Hanks et al. 1988). This motif has been suggested to play an important role in ATP binding (Taylor et al. 1992). The ku1 mutation might therefore alter the ATP-binding properties of the sur-1 gene product and cause a reduction in protein kinase activity.
Functional homology between Sur-1 MAP kinase and mammalian MAP kinases

Because the sur-1 gene product is structurally similar to mammalian p42 MAP kinases, we performed a functional homology assay by introducing a rat ERK2 gene (Boulton et al. 1991), which has a nearly identical protein sequence to that of human ERK2 (Owaki et al. 1992; Fig. 5 ), into $C$. elegans to see whether it could complement sur-1(ku1). A cDNA clone of the rat ERK2 gene (a gift from $M$. Cobb/ was fused to the sur-1 regulatory region at the beginning of the methionine codon in exon 2 . In addition, a $C$. elegans 3 ' polyadenylation signal sequence (from the unc-54 gene; A. Fire et al. in prep.) was added 
TG ATG CCA ACG TGG ATA CCT AAC AAT TTT TTC GCA CAG CCG ACA ACG AGA AAT GCT AAA CCA CCA TCG AAC GGG CAT CCA CAA GCG ACA CAA CAA CAA Met Pro Thr Trp Ile Pro Asn Asn Phe Phe Ala Gln Pro Thr Thr Arg Asn Ala Lys Pro Pro Ser Asn Gly His Pro Gin Ala Thr Gln Gln Gln

TCT GCT CCG GGA TCA TTG GCA TAC CGT AAT TCG AGC AAC ATA CCG AAT GGA GCA ACC AAC CAC GTC AGA CAA CAG AAA TGG CAG TAC ACT CGT TCA GGA 33 Ser Ala pro Giy Ser Leu Ala Tyr Arg Asn Ser Ser Asn Ile pro Asn Gly Ala Thr Asn His Val Arg Gln Gln Lys Trp Gln Tyr Thr Arg Ser Gly 198 CAT AGA AAG ATG GCC GAC GGA GAA GCG GTT ATC TCG ACG GTC Aac AAT GTC GAG GAA GTT CAT GGG CAA CTT TTT GAG GTT GCT CCC CGT TAT GTG AAT 66 His Arg Lys Met Ala Asp Gly Glu Ala Val Ile Ser Thr Val Asn Asn Val Glu Glu val his Gly Gln Leu Phe Glu Val Ala Pro Arg Tyr Val Asn T(ku1) GAT GA GGT GCT TAC GCG ATG GTC GC TCT GCA CTT GAC ACA ATm ACT CGT GAT CGC GTT GCT ATC AAA AAG ATT TCT CCA TTC GAA 99 Leu Ser Tyr Ile Gly Glu Gly Ala Tyr Gly Met Val Ala ser Ala Leu Asp Thr Ile Thr Arg Asp Arg val Ala Ile Lys Lys Ile Ser pro Phe Glu 396 CAT CAG ACA TTC TGT CAA CGG ACA CTT CGG GAA ATC AAA ATT CTT AAT CGA TTC AAG CAT GAG AAT ATC ATC AAT ATT CAA GAA ATC ATC CGT TCG GAG 132 His Gln Thr phe Cys Gln Arg Thr Leu Arg Glu Ile tys Ile Leu Asn Arg Phe Lys His Glu Asn Ile Ile Asn Ile Gln Glu Ile Ile Arg Ser Glu 495 ACT GTC GAT AGT TTG AAG GAT ATT 165 Thr Val Asp Ser Leu Lys Asp Ile Tyr Ile Val Gln Cys Leu Met Glu Thr Asp Leu Tyr Lys Leu Leu Lys Thr Gln Lys Leu Ser Asn Asp His Val

594 TGC TAC TTT CTC TAT CAA ATT CTC CGT GGT CTC AAA TAC ATT CAC TCT GCA AAT GTG CTC CAT CGT GAT TTG AAA CCA TCG AAT TTG TTM CTC AAC ACC 198 Cys Tyr Phe Leu Tyr Gln Ile Leu Arg Gly Leu Lys Tyr Ile His Ser Ala Asn Val Leu His Arg Asp Leu Lys pro Ser Asn Leu Leu Leu Asn Thr

693 ACA TGT GAT CTC AAA ATT TGC GAT TTT GGA TTG GCA CGT GTC ACC GAC CCA CAA ACC GAT CAT ACT GGG T"TC TTG ACC GAG TAT GTG GCT ACT CGT TGG 231 Thr Cys Asp Leu Lys Ile Cys Asp Phe Gly Leu Ala Arg Val Thr Asp Pro Gln Thr Asp His Thr Gly Phe Leu Thr Glu Tyr Val Ala Thr Arg Trp

792 TAT AGA GCT CCA GAG ATT ATG CTC AAT TCA AAG GGA TAC ACT AAA TCG ATT GAT GTC TGG TCT GTC GGA TGT ATT CTC GCA GAA ATG CTC AGT AAT CGC 264 Tyr Arg Ala pro Glu Ile Met Leu Asn Ser Lys Gly Tyr Thr Lys Ser Ile Asp Val Trp Ser Val Gly Cys Ile Leu Ala Glu Met Leu Ser Asn Arg

891 CCA TTG TTC CCG GGA AAA CAT TAT TTG GAT CAA CTC AAT TTG ATT TTG GCA GTT GTT GGA TCC CCG TCA AAT GCT GAT TTA CAA TGT ATT ATT AAT GAT 297 Pro Leu Phe Pro Gly Lys His Tyr Leu Asp Gln Leu Asn Leu Ile Leu Ala val val Gly Ser Pro Ser Asn Ala Asp Leu Gln Cys Ile Ile Asn Asp

990 AAg GCT CGA TCA TAT CTC ATT TCA TTG CCA CAC AAA CCA AAA CAA CCA TGG GCT CGG CTC TAT CCA GGA GCT GAT CCA AGA GCT CTT GAT TTA TTG GAC 330 Lys Ala Arg Ser Tyr Leu Ile Ser Leu Pro His Lys Pro lys Gln Pro Trp Ala Arg Leu Tyr pro Gly Ala Asp Pro Arg Ala Leu Asp Leu Leu Asp 7

1089 AAA ATG CTC ACT TTC AAT CCA CAT AAT CGT ATC GAC ATC GAG CAA GCA TTG GCT CAC CCA TAC TTG GAG CAA TAC TAC GAT CCA GGA GAT GAG CCA GTT 363 Lys Met Leu Thr phe Asn Pro His Asn Arg Ile Asp Ile Glu Gln Ala Leu Ala His Pro Tyr Leu Glu Gln Tyr Tyr Asp Pro Gly Asp Glu Pro Val

1188 TGT GAG GAA CCA TTC ACT TTG GAA ATG GAA TTC GAC GAT TTA CCG AAG GAG AAG CTG AAG GAG CTG ATT TGG GAA GAA GCC GAG GCT CAT CAC AGA CGA 396 Cys Glu Glu pro phe Thr Leu Glu Met Glu phe Asp Asp Leu Pro lys Glu Lys Leu Lys Glu Leu Ile Trp Glu Glu Ala Glu Ala His His Arg Arg

1287 ATG GAG GCA GAA GCG GCT GCA AGG AAT AAT GGA GGG CAG AAT CCT GTT TAG ATT AGT AGT ATT TAC CCA CTA AAT TAGTTATTTTTTCCACTTTTTTTTTATTTTC 429 Met Glu Ala Glu Ala Ala Ala Arg Asn Asn Gly Gly Gln Asn pro val Amb * * * * * * * $*$ AmB

1393 CACTAAGATTTTGGCATTTCAGTTTCTTTTTCGATGTATCATAATCCACTTCAAAACTCGATCGTTTAGTTGTTAGTAACCCCCCGTTCCCGCTGAAAAGCTTGTTGAAAATTTTCTAATATTTTACGTAT 1524 TTTGTAATATATTCATCCCCCAAAAAATATATATGTATATCCATTCCAGCTGTGATGTGAATGCACCTCCTCCAATTAATCACTCATTAATTATCGATTAGAATTCTGTCAAATTTTTTGTATAGGAATTC 1655 GCAAAAATCCGCCCATACTTGACTCAATTCCAATCGGCATTTGAATTTTTTTCAAATATTTTTTTTCACACACAATTCCATCAGAATCCCGCTGCTCTCCCCGTTCCACGTTTCCATTTTCTTTTTTCAATAT 1786 TTTTCAAATTTCCATTCTTCTGAAAATTCCTCTTTTTGAAAAAACCAAAAAATAGTATAATGTTCATCCTATGTGGGATCTCCAATGTTTTTCGTCTCTGTCCACATGTCCTCTTCTCGTTCTCCCTGAT 1917 TATTCAGTTTECCCGTTTTTATTGATTTCGGTATATAAATTCAAATTTCCAGGAAAAAAAAAAAAAA 1983

Figure 4. Nucleotide sequence of a sur-1 cDNA clone and predicted amino acid sequence. Arrowheads indicate the position of introns, as determined by comparison with the genomic sequence. The intron sizes are indicated in Fig. 3 . The nucleotide change in the sur-1(ku1) mutant is depicted $(\mathrm{C} \rightarrow \mathrm{T}$ at nucleotide 320$)$. The poly(A) sequence of another cDNA clone was determined to be 404 nucleotides upstream of the $3^{\prime}$ end of cDNA clone displayed (ends at underlined ATCC). Putative polyadenylation signal sequences (T. Blumenthal, pers. comm.), located 14 and 15 nucleotides upstream of the poly(A) sequences of two cDNA clones, are also marked (boldface). cDNAs that contain a sequence homologous to the C. elegans trans-splicing leader sequence SLl (Krause and Hirsh 1987) were also detected by PCR amplification, and this sequence was found to linked to the beginning of the second exon.

to the $3^{\prime}$ end of the ERK2 cDNA. The fusion gene construct was injected into a strain with genotype sur1(ku1); let-60(n1046 gf). pRF4, containing a rol-6(d) gene, was coinjected as a marker. The transgenic animals displayed a highly penetrant Muv phenotype $(>90 \%$; see Materials and methods), indicating that the rat ERK2 gene can functionally complement the sur-1 gene in $C$. elegans. The mammalian ERK2 proteins are thus able to interact with factors acting upstream and downstream of the sur-1 gene product. The structural and functional homology between the $C$. elegans Sur-1 MAP kinase and rat ERK2 suggests that the Sur-1 MAP kinase possesses all of the key biochemical properties identified for mammalian p42 MAP kinases (Anderson et al. 1990; Boulton et al. 1991; Payne et al. 1991). This result provides further in vivo evidence that the Ras-mediated signal transduction pathways are extremely conserved between worm and human.
Genetic interactions between sur-1(kul) and mutations in lin-15 or lin-1

To study the genetic interactions between sur- 1 and other genes in the vulval signaling pathway, double mutants were constructed with sur-1(ku1) and Muv loss-offunction mutations in either lin-15 or lin-1. The Muv phenotype of lin-15(n765) was suppressed by the sur1(ku1) allele (Table 1), consistent with the idea that sur-1 acts downstream of let-60 ras in the signaling pathway. In contrast, the Muv phenotype of $\operatorname{lin}-1$ (e1275) was not suppressed by the sur-1(ku1) mutation (Table 1), indicating that sur-1 acts before lin-1 in the signaling pathway. The genetic interactions of sur-1 with lin-15, let-60, and lin-1 are similar to those of the lin-45 gene, which encodes a homolog of the Raf proto-oncoprotein (Han et al. 1993). The relationship between sur-1 and lin-45 raf has not yet been established. However, there is substan- 


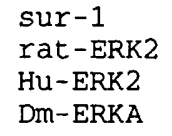

\section{sur-1}

rat-ERK2

$\mathrm{Hu}$-ERK2

DM-ERK-A

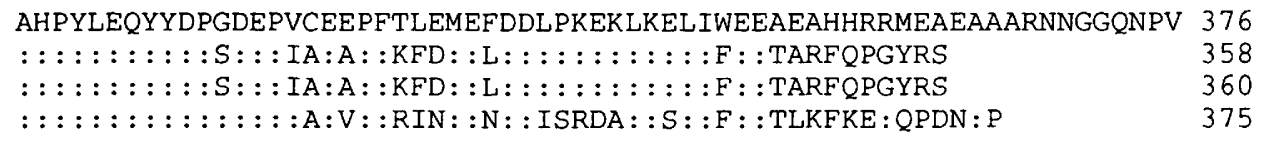

I MADGEAVISTVNNVEEVHGQLFEVAPRYVNLSYIGEGAYGMVASALDTITRDRVA 55 MAAAAAAGP $: M: R:: V: D: G::: I:::::::::::::$ C: :Y:NLNKV::: 50

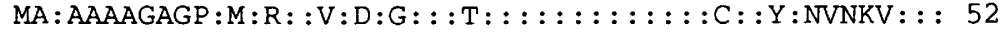
MEEFSSGSVVNGTGSTEVPQS:A:VIR: : I: : :G: : : IK:A: : : : : : : : :V::D:TLTNQ: : : 64

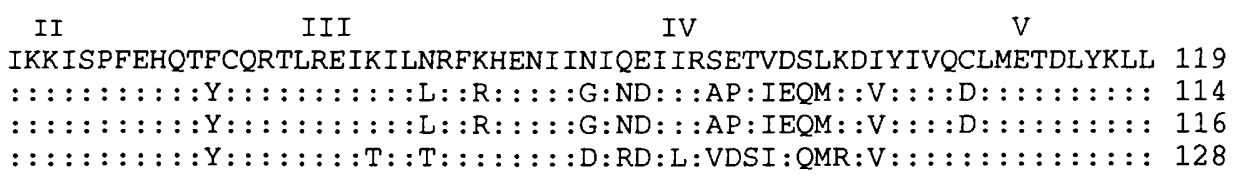
VI $\quad$ VII

KTQKLSNDHVCYFLYQILRGLKYIHSANVLHRDLKPSNLLLNTTCDLKICDFGLARVTDPQTDH 183

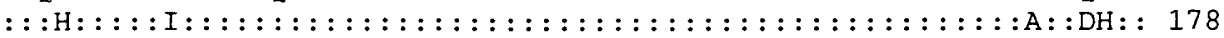

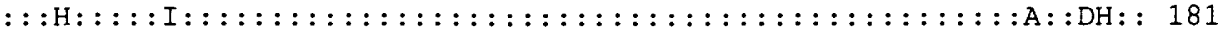

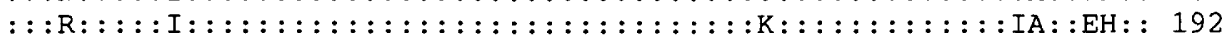

VIII IX TGFLTEYVATRWYRAPEIMLNSKGYTKSIDVWSVGCILAEMLSNRPLFPGKHYLDQLNLILAVV 247

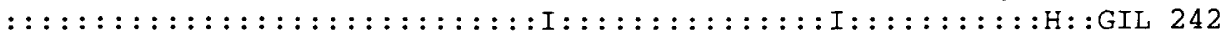

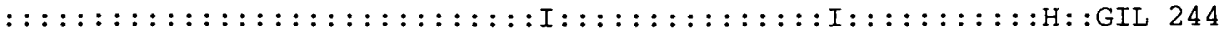

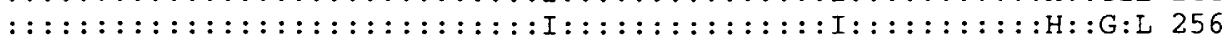

$\mathrm{X}$

$X I$ GSPSNADLQCIINDKARSYLISLPHKPKQPWARLYPGADPRALDLLDKMLTFNPHNRIDIEQAL 311 $:::: \mathrm{QE}:: \mathrm{N}:::: \mathrm{L}::: \mathrm{N}:: \mathrm{L}:::::: \mathrm{N}: \mathrm{V}:: \mathrm{N}:: F: \mathrm{N}:: \mathrm{SK}:::::::::::::::: \mathrm{K}:: \mathrm{EV}::::: 306$ $:::: Q E:: N:::: L::: N:: L::::: N: V:: N:: F: N:: S K::::::::::::::$ K::EV:::: 308 $:::: R D:: E:::: E::: N:: E::: F:: N V::: K: F: N:: A L::::: G:::::::: K:: P V: E:: 320$

Figure 5. Comparison of the predicted amino acid sequence encoded by sur-1 with that of the rat and human ERK2 proteins (Boulton et al. 1991; Owaki et al. 1992) and the Drosophila Dm ERK-A protein (Biggs and Zipursky 1992). Identical amino acids are indicated by colons. Roman numerals above the sequence denote kinase subdomains (Hanks et al. 1988).

tial evidence from studies in other organisms that MAP kinases are downstream targets of MAP kinase kinases (MKKs or MEKs), which in turn are the targets of Raf kinases (for review, see Ahn et al. 1992; Roberts 1992; Crews and Erikson 1993). Therefore, we propose that Sur-1 MAP kinase acts downstream of Lin-45 Raf kinase but before the lin-1 gene product in controlling vulval cell differentiation (Fig. 6).

\section{Discussion}

We have described the isolation and characterization of a mutation in the C. elegans gene sur-1 and have shown that the gene encodes a homolog of mammalian MAP kinases. The role of the MAP kinase homolog in the vulval signal transduction pathway is indicated by the suppression of the Muv phenotype of an activated let-60

Figure 6. Proposed functional relationships between sur-1 and other genes in the $C$. elegans vulval induction pathway. Arrows indicate positive regulation of one gene product by another. Neither arrows nor $\mathrm{T}$ bars necessarily reflect direct interactions. The lin-3 gene encodes a growth factor (GF)

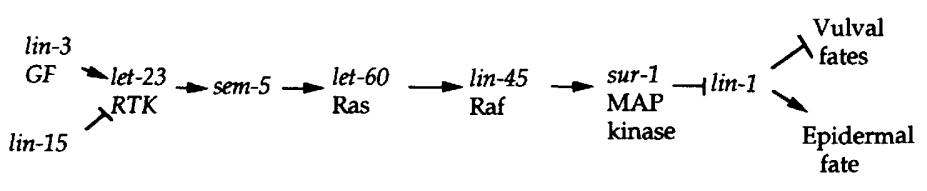
that is likely to be the inductive signal from the gonadal anchor cell (Hill and Sternberg 1992), whereas the let-23 gene product, a receptor tyrosine kinase (RTK) (Aroian et al. 1990), is likely to be the receptor for the signal. lin-15 is required for the inhibitory signal that is also upstream of let-23 (Ferguson et al. 1987; Herman and Hedgecock 1990). sem-5 encodes a protein with SH2 and SH3 domains and is likely to act between let-23 and let-60 ras (Clark et al. 1992). lin-45, which encodes a homolog of mammalian Raf kinases, is likely to act between let-60 ras and sur-1. lin-1 acts at the last step of the depicted pathway. The pathway as drawn does not include many other genes characterized to function in vulval fate specification (Ferguson et al. 1987; Kim and Horvitz 1990; Miller et al. 1993). For example, lin-31, which encodes a transcription factor likely to act after let-60 ras, appears to be required for proper expression of both vulval and epidermal cell fates by the six vulval precursor cells (Miller et al. 1993). 
ras mutation by the sur-1(ku1) mutation and by the reduction of vulval differentiation in some of the sur-1 mutants (Fig. 1). Our genetic data suggest that sur-1(ku1) causes a reduction of gene function. Null mutations in sur-1 may have a more severe mutant phenotype, such as early larval lethality, which is only partially associated with the sur-1(ku1) allele. Because vulval development is induced in the L3 larval stage, a completely penetrant Vul phenotype of a sur-1 null mutation might not be observed and isolated in initial genetic screens (e.g., Ferguson and Horvitz 1985). Alternatively, Sur-1 MAP kinase may be required only partially for the Let-60 Rasmediated vulval signaling pathway; there might be other MAP kinase-like proteins that provide redundant functions. However, in either case, complete suppression of the Muv phenotype of an activated let-60 ras mutation by the sur-1 mutation suggests that the sur-1(ku1) mutation significantly reduces the activity of the signaling pathway downstream of Ras.

It has been shown previously that the let-60(n1046 gf) mutation causes a Muv phenotype that is epistatic to elimination of the inductive signal (by ablating the gonadal anchor cell) or to loss-of-function mutations in the let-23 gene, which is likely to encode the receptor for the signal (Han et al. 1990). Such a Muv phenotype suggests that each of the six VPCs can, at least to some extent, activate the Ras-mediated signaling pathway in the absence of the upstream signal. Because the majority of the sur-1; let-60 double-mutant animals $(90 \%$; Table 1$)$ have a wild-type vulval cell lineage (P5.p-P7.p were induced to vulval tissue), the proper response of the VPCs to the signal from the gonadal anchor cell appears to be restored by reducing Sur-1 MAP kinase activity downstream of Let-60 Ras. This phenomenon may be explained as follows. In wild-type animals, the Let-60 Ras activity in P3.p, P4.p, and P8.p, which are not induced to generate vulval cells, is significantly lower than that in P5.p-P7.p, which are induced by the anchor cell signal to generate vulval cells. The let-60(n1046 gf) mutation elevates the let-60 ras activity in P3.p, P4.p, and P8.p so that these VPCs can generate vulval cells. However, the let $-60 \mathrm{mu}$ tation also elevates the Let-60 Ras activity in P5.p-P7.p so that they still have significantly higher let-60 ras activity than that in P3.p, P4.p, and P8.p. In the sur-1(ku1); let-60(n1046 gf) double mutant, the sur-1 mutation may reduce the signaling activity in all six VPCs so that they have near wild-type signaling outputs. Because the vulval differentiation in the sur-1 single mutant is similar to that of the double mutant (mostly wild type), the reduction of the signaling activity by the sur-1 mutation must be limited because P5.p-P7.p still have enough MAP kinase activity to specify vulval cell fates in the sur-1 single mutant. In other words, in most of the sur-1(ku1); let-60(n1046 gf) double-mutant animals, the signaling output of P.3p, P4.p, and P8.p is below a "vulva-inducing threshold," wheres in most of the sur-1 single-mutant animals, the signaling output of P.5.p-P7.p is above the vulva-inducing threshold.

ERKs are serine/threonine protein kinases that are activated in response to a wide variety of extracellular stimuli (Cobb et al. 1991; Pelech and Sanghera 1992; Thomas 1992). They have been characterized as key components acting at a relatively late step in different types of signal transduction pathways. For example, MKKs or MEKs, which are the activators of MAP kinases, have been shown to be the targets of Raf kinases (Dent et al. 1992; Howe et al. 1992; Kyriakis et al. 1992), MEK kinases (MEKKs) (Lange-Carter et al. 1993), or c-Mos (Posada et al. 1993). Each of these upstream components also plays roles in multiple cell signaling processes. Recent studies in Drosophila have also shown that a MAP kinase, encoded by the rolled/Dm-ERK-A gene, is required for multiple receptor tyrosine kinasesmediated signaling pathways controlling cell differentiations (L. Zipursky and E. Hafen, pers. comm.). Therefore, MAP kinases appear to be central components acting in a wide range of cellular processes, including cell proliferation, differentiation, and secretion. As a wellestablished genetic organism to study animal development, C. elegans provides us with distinct advantages to analyze specific functions of a common signaling molecule that can interact with many upstream and downstream factors. As indicated in the results of this study, the sur-1 gene is expected to play roles in many developmental processes in C. elegans. Besides the effect on vulval cell differentiation, the sur-1 mutation also results in larval lethality and a cold-sensitive egg-laying defect that is independent of the extent of vulval cell differentiation. Understanding the causes of these defects should help us to understand the role of MAP kinases in specific cell signaling pathways controlling specific developmental events.

The study of the sur-1 gene demonstrated that suppressor analysis is a very effective way to identify components in a specific cell signaling pathway. The sur1(ku1) mutation is just one of many suppressors of a hyperactive let-60 ras mutation isolated in our laboratory. Genetic and molecular analysis of genes defined by these suppressors may reveal roles of proteins with or without known biochemical identities in Ras-mediated signaling pathways controlling cell differentiation and proliferation.

\section{Materials and methods}

\section{Strains and genetic methods}

Methods for the culturing, handling, and genetic manipulation of C. elegans were as described previously (Brenner 1974; Wood et al. 1988). All genetic experiments were performed at $20^{\circ} \mathrm{C}$, or at $15^{\circ} \mathrm{C}$ as specified. Methods for anlysis of vulval defects under dissecting microscope were as described previously (Sulston and Horvitz 1977; Han et al. 1990|. Nomarski microscopy and photography were performed as described previously (Sternberg and Horvitz 1986; Avery and Horvitz 1987). The references for alleles of various mutants used in this study are let-60(n1046 gf) and lin-15(n765) (Ferguson and Horvitz 1985); dpy-20(e1282) (Hosono et al. 1982); lin-1(e1275) (Horvitz and Sulston 1980); unc-93(e1500) and nDf11 (Greenwald and Horvitz 1980); unc32(e189), unc-36(e251), and dpy-17(e164) (Brenner 1974); him5(e1490) and him-8(e1489) (Hodgkin et al. 1979); unc-79(e1068) (Hodgkin 1983); $d p y-27(y 169)$ and yDf10 (Plenefisch et al 
1989); ncl-1(e1865) (Austin and Kimble 1987; E. Hedgecock, pers. comm.); MJ\#NEC2 (Nishiwaki et al. 1993); qC1 (J. Kimble, pers. comm.); and $s D p 3$ (Rosenbluth et al. 1985).

\section{Isolation and genetic characterization of the sur-1(kul) allele}

sur-1(ku1) was isolated as a recessive suppressor of the Muv phenotype of let-60(n1046 gf). Animals homozygous for let60(n1046 gf) were mutagenized with $50 \mathrm{~mm}$ ethylmethane sulfonate (EMS) (Brenner 1974), and F2 progeny were screened for non-Muv revertants. In many cases, candidates were picked with an Egl phenotype. Candidates that continued to segregate non-Muv progeny were characterized further. $k u 1$ is 1 of $>50$ suppressor mutations isolated after screening $>50,000$ mutagenized haploid genomes. Outcrossing and genetic mapping of ku1 were performed using standard genetic procedures (Brenner 1974). Egg-laying ability and the extent of vulval differentiation were examined as described previously (Ferguson and Horvitz 1985; Han et al. 1990|. The percentage of lethality associated with sur-1(ku1) homozygotes was determined by collecting eggs and examining larval lethality in the following 2 days.

To determine the phenotype of sur-1/(ku1)/deficiency, we attempted to construct a strain of $\operatorname{sur}-1(\mathrm{ku1}) / \mathrm{nDf} 11$. Five to ten sur-1(ku1) dpy-17(e164)/qC1; him-5(e1490) males were crossed with one to three $n D f 11+/$ unc-79(e1068) dpy-17(e164) hermaphrodites in individual plates. The crosses were performed in 34 plates with a total of 47 hermaphrodites. Because $n D f 11$ uncovers unc-79 but not dpy-17 (Fig. 3A), nDf11 + /unc-79 dpy17 animals are Unc non-Dpy. Non-Dpy, non-Unc F1 hermaphrodites were individually picked from each mating plate. The genotypes of these F1 animals were determined by examining the phenotypes of their progeny. Among 189 F1 hermaphrodites picked, 10 were sterile, 179 were unc-79 $d p y-17 / q C 1$ animals, and none were sur-1(ku1) $d p y-17 / n D f 11$. As a control, dpy-17/ $q C 1$; him-5 males were crossed with $n D f 11+/$ unc-79 dpy-17 hermaphrodites. Among 39 non-Dpy, non-Unc cross progeny obtained, 23 were unc-79 dpy-17/qC1 animals and 16 were $+d p y-17 / n D f 11$ + animals.

Strains that are homozygous for let-60(n1046 gf) and contain a duplication $(s D p 3)$ that covers sur-1 were constructed as follows. Males of genotype dpy-17(e164) ncl-1(e1865) unc36(e251); him-8(e1489); sDp3 were crossed with sur-1(ku1) dpy-17; let-60(n1046 gf) hermaphrodites. Phenotypically nonDpy Fl cross progeny, which should have genotype sur-1 dpy-17 $++/+$ dpy-17 ncl-1 unc-36; + let-60(gf) /him-8 +; sDp3, were picked individually. About 10 Muv F2 non-Dpy hermaphrodites were then picked individually to plates for homozygous let-60(gf) animals. The complete genotype of each F2 animal was determined by the phenotypes of F3 progeny. For example, strains of genotype sur-1 dpy-17/sur-1 dpy-17; let-60(gf)/let$60(\mathrm{gf})$; $s D p 3$ were obtained by determining that the F2 animals segregated no Dpy-Unc F3 progeny, the majority of non-Dpy F3 progeny were Muv, and none of the F3 Dpy animals were Muv. To determine the Muv percentage of sur- $1 /+/+$; let $-60(\mathrm{gf}) /$ let$60(g f) ; s D p 3$, L4 progeny of sur-1 dpy-17 $++/+d p y-17$ ncl-1 unc-36; let-60(gf)/let-60(gf); sDp3 animals were picked individually, and the phenotype (Muv or non-Muv) of each was scored on the next day. The genotype of each was determined by the phenotype of their progeny. Among 49 animals with genotype sur-1 dpy-17 + +/+ dpy-17 ncl-1 unc-36; let-60(gf)/let-60(gf); $s D p 3,47$ were Muv $(96 \%)$. A strain with genotype sur-1 $d p y-$ 17/sur-1 dpy-17; him-8/him-8; sDp3 was constructed by picking non-Muv non-Dpy hermaphrodites from sur-1 dpy-17+ $+/+$ dpy-17 ncl-1 unc-36; + let-60(gf) /him-8 +; sDp3 animals. The genotype of picked animals was determined by examining the phenotypes of their progeny.

\section{Genetic mapping of sur-1}

Two-factor and three-factor mapping using genetic markers were performed by standard methods (Brenner 1974). The results of three-factor mapping are listed in Table 3. sur-1 was also mapped to the right of unc- 79 by determining that $y D f 10$ uncovers unc- 79 but not sur-1. This was done by PCR amplification and sequence analysis of the sur-1 DNA-coding region from sur-1(ku1) dpy-17+/yDf10 + unc-32 heterozygous animals. We found that both wild-type and mutant DNAs were present in the DNA sample, indicating that the right breakpoint of yDf10 is located between unc-79 and the sur-1(ku1) mutant lesion. sur-1(ku1)/yDf10 animals are wild type in vulval development, whereas unc-79/yDf10 animals are Unc.

To map the sur-1 gene relative to the position of MJ\#NEC2 (a Tc1 insertion), we obtained the strain unc-79 M/\#NEC2 dpy-17 (Nishiwaki et al. 1993). The strain unc-79 + M/\#NEC2 dpy-17 $+1+$ sur- $1++$ unc-32 was constructed, and both Unc-79 nonDpy-17 and Dpy-17 non-Unc-79 recombinants were isolated. The Egl phenotype was scored in each recombinant to determine the presence of the sur-1 mutation. Sequence flanking MJ\#NEC2 (also a gift from J. Miwa) was used to probe DNA isolated from the recombinants to determine the presence of MJ\#NEC2. The result is listed in Table 3.

\section{Double-mutant construction}

The sur-1(ku1); let-60(n1046 gf) double mutant was reconstructed after the original sur-1 allele was outcrossed multiple times. To construct the sur-1; lin-15 double mutant, lin$15(n 765) / 0$ males were crossed to sur-1(ku1); dpy-20(e1282) hermaphrodites. Muv F2 animals were picked individually from F1 cross progeny that were sur $-1 /+;$ dpy-20/ + ; lin-15/ + Four of nine such Muv parents segregated non-Muv progeny that should have the genotype sur-1/sur-1; lin-15/lin-15. The sur-1; lin-1 double mutant was constructed by crossing lin-1(e1275) dpy-20(e1282)/ + + males to sur-1(ku1)/sur-1(ku1) hermaphrodites. Non-Muv, Egl F2 progeny were individually picked from $\mathrm{F} 1$ cross progeny that segregated Muv and Dpy animals (sur-1/t; lin-1 dpy-20/ + +1. Two of three of these F2 nonMuv, Egl animals have the genotype sur-1/sur-1; lin-1 $d p y$ $20 /++$. Muv Dpy animals that should have a genotype of sur-1; lin-1 dpy-20 were picked from progeny of $\mathrm{F} 2$ animals that segregated Muv and Dpy progeny.

\section{Microinjection transformation}

All cosmids used in this study were obtained from A. Coulson and J. Sulston (MRC Laboratory of Molecular Biology, Cambridge, UK). DNA isolation, analysis, and subcloning were performed by standard molecular biology methods. Microinjection of cloned DNAs into the gonadal syncytia of C. elegans hermaphrodites was carried out as described previously (Han and Sternberg 1990; Mello et al. 1991). All cosmids listed in Figure $3 \mathrm{~B}$ were initially injected as a pool of three nonoverlapping cosmids at a concentration of $\sim 20 \mu \mathrm{g} / \mathrm{ml}$. All positive clones and several negative clones were then injected individually. A strain of sur-1(ku1); let-60(n1046 gf) was used as the host strain for microinjection. The Muv phenotype of let-60(n1046 gf) $(\sim 95 \%)$ is completely suppressed in the double mutant. Rescue of the sur-1 mutant phenotype was indicated by reappearance of the Muv phenotype. Each injection was scored with at least two independent transgenic lines. We observed that the extent of rescue was not consistent among different transgenic lines obtained from injecting the same DNA clones. For example, among 18 transgenic lines obtained from injecting cosmid R74, 
9 produced a high percentage of Muv animals, whereas 9 produced mostly non-Muv animals. Among five transgenic lines derived from injecting pYW2 plus pYW7, four produced a high percentage of Muv animals, whereas one produced mostly nonMuv animals. The percentage of Muv animals in those positive transformant lines is usually $>90 \%$. The host strain appears to be sensitive to the dosage of the exogenous sur-1 gene because stable transgenic lines were not recovered following injections at $>30 \mu \mathrm{g} / \mathrm{ml}$. Even at $\sim 20 \mu \mathrm{g} / \mathrm{ml}$, transgenic lines were more difficult to obtain with positive clones than with negative clones. Transgenic animals with a Muv phenotype were often sick and failed to produce progeny. These results suggest that proper expression of the sur-1 gene on extrachromosomal DNA may be critical for its proper function. Similar results were observed in microinjection experiments with the let-60 ras gene and the lin-45 raf gene (Han and Sternberg 1990; Han et al. 1993). The Muv phenotype was never associated with transgenic lines obtained from the negative clones. For example, the Muv phenotype was never seen in 9 stable lines obtained from injecting C12E9 or in 13 stable lines obtained from injecting pYW2 alone.

\section{DNA sequence analysis}

cDNA and genomic DNA clones were sequenced by a doublestrand DNA sequencing method using Sequenase (U.S. Biochemical). Both cDNA and genomic DNA clones were sequenced using primers that hybridize to the polylinker region of the pBluescript $\mathrm{SK}|+|$ vector or by primers hybridizing to regions of sur-1 sequence. The 3 ' end of the cDNA sequences was determined by identifying the poly $(\mathrm{A})$ tails of the cDNA clones. The 3' end of another cDNA clone is $\sim 404$ bp shorter. Putative $C$. elegans polyadenylation signal sequences (T. Blumenthal, pers. comm.) were found 14 and 15 nucleotides upstream of the end of the two types of cDNA clones (Fig. 4). To determine the DNA lesion in sur-1(ku1), DNA fragments containing sur-1coding regions and intron/exon boundaries were obtained by PCR amplification from homozygous mutant animals as well as wild-type animals. These PCR fragments were gel purified and sequenced directly (Kretz et al. 1989). Sequence comparison was performed through the BLASTp program.

\section{Construction and analysis of a sur-1-ERK2 fusion gene}

The sur-1-ERK2 fusion plasmid (pYW42) was constructed by combining plasmid pMH203, which contains part of inton 1 of sur- 1 fused to the first 26 nucleotides of the rat ERK2 coding region, with pMH204, which contains the coding region of the rat ERK2 gene fused with the 3' end of the C. elegans unc-54 gene. pMH203 was constructed as follows. Two oligonucleotides (5'-GCTCAATAGTAATCTCCTAA and 5'-CTCCGGGCCCGCCGCCGCCGCCGCCGCCATCCCGTAAGCACCTTCTCC) were used as primers to PCR-amplify pYW2, which contains part of intron 1 and exon 2 of sur-1. The $5^{\prime}$ primer was derived from sur-1 sequence to the left of the HindIII site in intron 1 (Fig. 3C). The last 21 nucleotides of the 3 ' primer complement sur-1 sequence preceding and including the putative translational start codon. The rest of the oligonucleotide complements the rat ERK2-coding region from the translational start site. This short ERK2 sequence contains an ApaI recognition site. After 10 rounds of PCR amplification, the PCR products were digested with HindIII and ApaI. The resulting DNA fragments were ligated to the large HindIII-ApaI fragment of pYW2 to generate the plasmid pMH203. pMH204 was constructed as follows. pPD49.26 (a gift from A. Fire) was digested with ApaI. Klenow fragment of Escherichia coli DNA polymer- ase I was used to generate blunt ends, followed by digestion with Sall. An $\sim 760$-bp fragment containing the C. elegans unc54 poly(A) addition site was isolated from an agarose gel and cloned into NpT7-5.ERK2.L (containing the rat ERK CDNA; a gift from M. Cobb), which had been digested with HindIII, blunt-ended, and then digested with Sall. The resulting clone was digested with SalI, blunt-ended, digested with EcoRV, then self-ligated to generate plasmid $\mathrm{pMH} 204$. This procedure removed the NcoI site between Sall and EcoRV. The final plasmid, pYW42, was made by digesting pMH203 with ApaI and SmaI. The large fragment was isolated and cloned into pMH204, which was digested with $N c o I$, blunt-ended, and then digested with ApaI. The final plasmid, pYW42, was injected into sur1(ku1); let-60(n1046 gf) animals at $\sim 20 \mu \mathrm{g} / \mathrm{ml}$ (pRF4 was coinjected as a marker; see above). Among $\sim 269 \mathrm{Fl}$ transformants, 261 were Muv. About 40 of those Muv animals were either sick or dying at early adult stage. Among 13 stable transformant lines analyzed, 12 displayed a highly penetrant Muv phenotype $(>90 \%)$. As a control, pMH204, which contains only the rat ERK2 cDNA and the $C$. elegans unc-54 poly(A) addtion sequence, was injected, and no Muv phenotype was observed in the transgenic animals. This result indicates that the first intron of sur- 1 is required for the expression of the ERK 2 cDNA in C. elegans.

\section{Acknowledgments}

We thank J. Miwa for strains containing the Tcl RFLP (MJ\#NEC2) and a plasmid containing the DNA fragment flanking the Tc1, M. Cobb for the plasmid containing the rat Erk2 cDNA, A. Fire for plasmid pPD49.26, R. Barstead for the cDNA library, A. Coulson and the Medical Research Council group for cosmids, and B. Meyer, J. Thomas, W. Wood, and J. Yochem for various $C$. elegans strains. Some of the strains were provided by the Caenorhabditis Genetics Center, which is funded by the National Institutes of Health National Center for Research resources. We also thank Y. Han for technical assistance, N. Ahn, H. Chen, M. Hara, S. Mansour, Dan Nieuwlandt, M. Sundaram, K. Van Auken, and J. Yochem for comments, and members of our and W. Wood's laboratories for valuable suggestions and discussions during the course of this study. We also thank S. Kim, K. Kornfeld, L. Zipursky, and E. Hafen for communicating results prior to publication. Y. W. thanks Larry Gold for support. M. H. is a Lucille P. Markey Scholar, a Basal O'Connor Scholar of the March of Dime Foundation, and a Searle Scholar. This research has been supported by a U.S. Public Health Service grant (R01 GM47869) and partly by a grant from the Council for Tobacco Research to M.H.

The publication costs of this article were defrayed in part by payment of page charges. This article must therefore be hereby marked "advertisement" in accordance with 18 USC section 1734 solely to indicate this fact.

\section{Note added in proof}

The accession number for the sur-1 cDNA sequence (Fig. 4) is U03879.

\section{References}

Ahn, N.G., R. Seger, and E.G. Kreb. 1992. The mitogen-activated protein kinase activator. Curr. Opin. Cell Biol. 4: 992999.

Anderson, N.G., J.L. Maller, N.K. Tonks, and T.W. Sturgill. 1990. Requirement for integration of signals from two dis- 
tinct phosphorylation pathways for activation of MAP kinases. Nature 343: 651-653.

Aroian, R.V. and P.W. Sternberg. 1991. Multiple functions of let-23, a Caenorhabditis elegans receptor tyrosine kinase gene required for vulval induction. Genetics 128: 251-267.

Aroian, R.V., M. Koga, J.E. Mendel, Y. Ohshima, and P.W. Sternberg. 1990. The let-23 gene necessary for Caenorhabditis elegans vulval induction encodes a tyrosine kinase of the EGF receptor subfamily. Nature 348: 693-699.

Austin, J. and J. Kimble. 1987. glp-1 is required in the germ line for regulation of the decision between mitosis and meiosis in C. elegans. Cell 51: 589-599.

Avery, L. and H.R. Horvitz. 1987. A cell that dies during wildtype $C$. elegans development can function as a neuron in a ced-3 mutant. Cell 51: 1071-1078.

Barstead, R.J. and R.H. Waterston. 1989. The basal component of the nematode dense-body is vinculin. I. Biol. Chem. 264: 10177-10185.

Beitel, G., S. Clark, and H.R. Horvitz. 1990. The Caenorhabditis elegans ras gene let- 60 acts as a switch in the pathway of vulval induction. Nature 348: 503-509.

Biggs, W.H.I. and S.L. Zipursky. 1992. Primary structure, expression, and signal-dependent tyrosine phosphorylation of Drosophila homolog of extracellular signal-regulated kinases. Proc. Natl. Acad. Sci. 89: 6295-6299.

Boulton, T.G., S.H. Hye, D.J. Robbins, N.Y. Ip, E. Radziejewska, S.D. Morgenbesser, R.A. DePinho, N. Panayotatos, M.H. Cobb, and G.D. Yancopoulos. 1991. ERKs: A family of protein-serine/threonine kinases that are activated and tyrosine phosphorylated in response to insulin and NGF. Cell 65: 663-675.

Brenner, S. 1974. The genetics of Caenorhabditis elegans. Genetics 77: 71-94.

Clark, S.G., M.J. Stern, and H.R. Horvitz. 1992. C. elegans cellsignalling gene sem -5 encodes a protein with $\mathrm{SH} 2$ and $\mathrm{SH} 3$ domain. Nature 356: 340-344.

Cobb, M.H., T.G. Boulton, and D.J. Robbins. 1991. Extracellular signal-regulated kinases-ERKs in progress. Cell Regul. 2: 965-978.

Crews, C.M. and R.L. Erikson. 1993. Extracellular signals and reversible protein phosphorylation: What to mek of it all. Cell 74: 215-217.

Dent, P., W. Haser, T.A.J. Haystead, L.A. Vincent, T.M. Roberts, and T.W. Sturgill. 1992. Activation of mitogen-activated protein kinase kinase by $\mathrm{v}$-Raf in NIH $3 \mathrm{~T} 3$ cells and in vitro. Science 257: 1404-1407.

Ferguson, E. and H.R. Horvitz. 1985. Identification and characterization of 22 genes that affect the vulval cell lineages of Caenorhabditis elegans. Genetics 110: 17-72.

Ferguson, E.L., P.W. Stemberg, and H.R. Horvitz. 1987. A genetic pathway for the specification of the vulval cell lineages of Caenorhabditis elegans. Nature 326: 259-267.

Greenwald, I.S. and H.R. Horvitz. 1980. unc-93(e1500): A behavioral mutant of Caenorhabditis elegans that defines a gene with a wild-type null phenotype. Genetics 96: 147-164.

Han, M. and P.W. Stemberg. 1990. let-60, a gene that specifies cell fates during $\mathrm{C}$. elegans vulval induction, encodes a ras protein. Cell 63: 921-931.

Han, M., R. Aroian, and P.W. Sternberg. 1990. The let-60 locus controls the switch between vulval and non-vulval cell types in C. elegans. Genetics 26: 899-913.

Han, M., A. Golden, Y. Han, and P.W. Sternberg. 1993. C. elegans lin-45 raf gene participates in let-60 ras-stimulated vulval differentiation. Nature 363: 133-140.

Hanks, S.K., A.M. Quinn, and T. Hunter. 1988. The protein kinase family: Conserved features and deduced phylogeny of the catalytic domains. Science 241: 42-52.

Herman, R.K. and E.M. Hedgecock. 1990. Limitation of the size of the vulval primordium of Caenorhabditis elegans by lin15 expression in surrounding hypodermis. Nature 348: 169171.

Hill, R.J. and P.W. Sternberg. 1992. The gene lin-3 encodes an inductive signal for vulval development in C. elegans. $\mathrm{Na}$ ture 358: 470-476.

Hodgkin, J. 1983. Male phenotypes and mating efficiency in Caenorhabditis elegans. Genetics 103: 43-64.

Hodgkin, J., H.R. Horvitz, and S. Brenner. 1979. Nondisjunction mutants of the nematode Caenorhabditis elegans. Genetics 91: 67-94.

Horvitz, H.R. and P.W. Sternberg. 1991. Multiple intercellular signalling systems control the development of the C. elegans vulva. Nature 351: 535-541.

Horvitz, H.R. and J.E. Sulston. 1980. Isolation and genetic characterization of cell-lineage mutants of the nematode Caenorhabditis elegans. Genetics 96: 435-454.

Hosono, R., K. Hirahara, S. Kuno, and T. Kurihara. 1982. Mutants of Caenorhabditis elegans with Dumpy and Rounded head phenotype. J. Exp. Zool. 235: 409-421.

Howe, L.R., S.J. Leevers, N. Gomez, S. Nakielny, P. Cohen, and C. Marshall. 1992. Activation of the MAP kinase pathway by the protein kinase raf. Cell 71: 335-342.

Kim, S.K. and H.R. Horvitz. 1990. The Caenorhabditis elegans gene lin-10 is broadly expressed while required specifically for the determination of vulval cell fates. Genes \& Dev. 4: 357-371.

Krause, M. and D. Hirsh. 1987. A trans-spliced leader sequence on actin mRNA in Caenorhabditis elegans. Cell 49: 753 761.

Kretz, K.A., G.S. Carson, and J.S. O’Brien. 1989. Direct sequencing from low-melt agarose with Sequenase. Nucleic Acids Res. 17: 5864.

Kyriakis, J.M., H. App, X.-F. Zhang, P. Banerjee, D.L. Brautigan, U.R. Rapp, and J. Avruch. 1992. Raf-1 activates MAP kinasekinase. Nature 358: 417-421.

Lange-Carter, C.A., C.M. Pleiman, A.M. Gardner, K.J. Blumer, and G.L. John. 1993. A divergence in the MAP kinase regulatory network defined by MEK kinase and Raf. Science 260: 315-319.

Mello, C.C., J.M. Kramer, D. Stinchcomb, and V. Ambros. 1991. Efficient gene transfer in C. elegans after microinjection of DNA into germline cytoplasm: Recombination drives the assembly of heritable transgenic structures. EMBO /. 10: 3959-3970.

Miller, L.M., M.E. Gallegos, B.A. Morisseau, and S. Kim. 1993. Lin-31, a Caenorhabditis elegans HNF-3/fork head transcription factor homolog, specifies three alternative cell fates in vulval development. Genes \& Dev. 7: 933-947.

Nishiwaki, K., T. Sano, and J. Miwa. 1993. emb-5, a gene required for the correct timing of gut precursor cell division during gastrulation in Caenorhabditis elegans, encodes a protein similar to the yeast nuclear protein SPT6. Mol. \& Gen. Genet. 239: 313-322.

Owaki, R. Markar, T.G. Boulton, M.H. Cobb, and T.D. Geppert. 1992. Extracellular signal-regulated kinases in T cells: Characterization of human ERK1 and ERK2 cDNAs. Biochem. Biophys. Res. Commun. 182: 1416-1422.

Payne, D.M., A.J. Rossomando, P. Martino, A.K. Erickson, J.-H. Her, J. Shabanowitz, D.F. Hunt, M.J. Weber, and T.W. Sturgill. 1991. Identification of the regulatory phosphorylation sites in pp42/mitogen-activated protein kinase (MAP kinase). EMBO I. 10: 885-892.

Pelech, S.L. and J. Sanghera. 1992. Mitogene-activated protein 
kinases versatile transducers for cell signaling.

Plenefisch, J.D., L. DeLong, and B.J. Meyer. 1989. Genes that implement the hermaphrodite mode of dosage compensation in Caenorhabditis elegans. Genetics 121: 57-76.

Posada, J., N. Yew, N.G. Ahn, G.F. Vande Woude, and J.A. Cooper. 1993. Mos stimulates MAP kinase in Xenopus oocytes and activates a MAP kinase kinase in vitro. Mol. Cell. Biol. 13: 2546-2553.

Roberts, T.M. 1992. A signal chain of events. Nature 360: 534535.

Rosenbluth, R.E., C. Cuddeford, and D.L. Baillie. 1985. Mutagenesis in Caenorhabditis elegans. II. A spectrum of mutational events induced with $1500 \mathrm{R}$ of gamma-radiation. Genetics 109: 493-511.

Sternberg, P.W. and H.R. Horvitz. 1986. Pattern formation during vulval development in Caenorhabditis elegans. Cell 44: 761-772.

Sulston, J. and H.R. Horvitz. 1977. Postembryonic cell lineages of the nematode Caenorhabditis elegans. Dev. Biol. 56: 110156.

Taylor, S.S., D.R. Knighton, J. Zheng, L.F. Ten Eyck, and J.M. Sowadaski. 1992. Structural framework for the protein kinase family. Annu. Rev. Cell Biol. 8: 429-462.

Thomas, G. 1992. MAP kinase by any other name smells just as sweet. Cell 68: 3-6.

Wood, W.B. and the Community of C. elegans Researchers. 1988. The nematode Caenorhabditis elegans. Cold Spring Harbor Laboratory, Cold Spring Harbor, New York. 


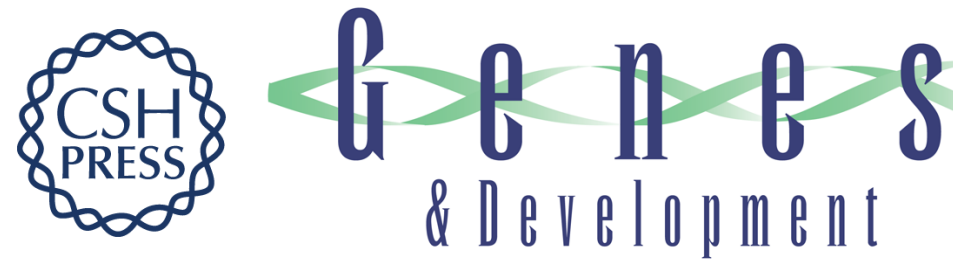

\section{Suppression of activated Let-60 ras protein defines a role of Caenorhabditis elegans Sur-1 MAP kinase in vulval differentiation.}

Y Wu and $\mathrm{M}$ Han

Genes Dev. 1994, 8:

Access the most recent version at doi:10.1101/gad.8.2.147

References This article cites 48 articles, 17 of which can be accessed free at: http://genesdev.cshlp.org/content/8/2/147.full.html\#ref-list-1

License

Email Alerting

Receive free email alerts when new articles cite this article - sign up in the box at the top Service right corner of the article or click here.

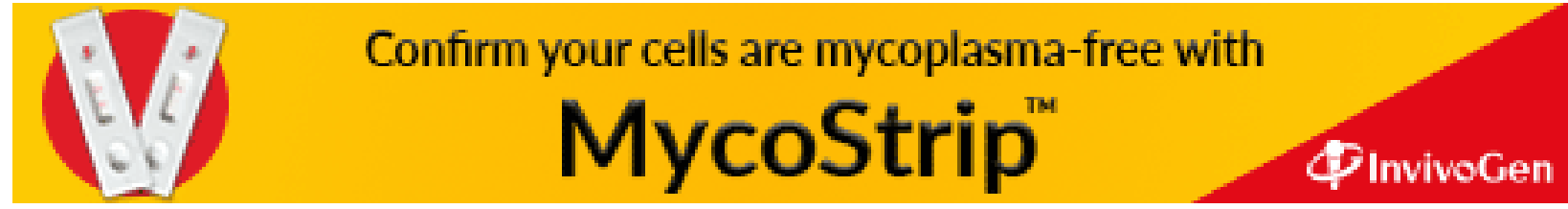

